\title{
Global existence for the spherically symmetric Einstein-Vlasov system with outgoing matter
}

\author{
Håkan Andréasson \\ Department of Mathematics, Chalmers, \\ S-41296 Göteborg, Sweden \\ email: hand@math.chalmers.se \\ Markus Kunze \\ Fachbereich Mathematik \\ Universität Duisburg-Essen \\ D-45117 Essen, Germany \\ email: markus.kunze@uni-due.de \\ Gerhard Rein \\ Fakultät für Mathematik und Physik \\ Universität Bayreuth, \\ D-95440 Bayreuth, Germany \\ email: gerhard.rein@uni-bayreuth.de
}

February 4, 2018

\begin{abstract}
We prove a new global existence result for the asymptotically flat, spherically symmetric Einstein-Vlasov system which describes in the framework of general relativity an ensemble of particles which interact by gravity. The data are such that initially all the particles are moving radially outward and that this property can be bootstrapped. The resulting non-vacuum spacetime is future geodesically complete.
\end{abstract}




\section{Introduction}

Global properties of non-vacuum spacetimes are one of the central themes of the mathematical analysis of general relativity. A matter model which has proven particularly suitable for such an analysis is the collisionless gas as described by the Vlasov equation. Here the matter content of spacetime is represented by a large ensemble of particles with a density function $f$ on phase space, i.e., on the mass shell of the tangent bundle of the spacetime manifold. The particles move along time-like geodesics which is equivalent to $f$ satisfying the corresponding continuity equation, i.e., the Vlasov equation, and the energy momentum tensor is then expressed in terms of $f$.

For a general introduction to the resulting Einstein-Vlasov system we refer to [1] and [10]. A major result for the asymptotically flat case of this system is a global existence result for small, spherically symmetric initial data [8. In addition, there is numerical evidence [2, 5, 9] that at least in the spherically symmetric case the weak cosmic censorship conjecture holds: The gravitational collapse of regular initial data leads to a black hole rather than a naked singularity.

In view of the small data result mentioned above and the lack of proof of global existence for general data (in Schwarzschild coordinates or in maximalareal coordinates) it is natural to ask if one can find an open set of initial data which are not small in the sense of [8] but for which global existence holds. In the present paper we do find initial data with this property by establishing global existence for data which are such that initially all the particles in the ensemble move radially outward sufficiently fast so that they can be shown to keep on moving outward for all future time. This configuration results in estimates on the geometry of spacetime which imply that it is future geodesically complete.

The basic mechanism of the proof is completely different from the one for small initial data which relied on the dispersive properties of the Vlasov matter model when the fields are small. Since its actual implementation for the Einstein-Vlasov system quite hides this mechanism, it should be useful to explain it first in the case of the much simpler Vlasov-Poisson system which is the Newtonian limit of the Einstein-Vlasov system. Due to the spherical symmetry the maximal force experienced by a particle at distance $r$ from the origin is $-M / r^{2}$ in the Vlasov-Poisson case, where $M>0$ is the total mass 
of the ensemble. Hence along any particle trajectory

$$
\frac{d}{d t}\left(\frac{1}{2} w^{2}-\frac{M}{r}\right)=w \dot{w}+\frac{M}{r^{2}} \dot{r}=w\left(\dot{w}+\frac{M}{r^{2}}\right) \geq 0,
$$

as long as its radial velocity $\dot{r}=w=x \cdot v / r \geq 0$. Hence

$$
\frac{1}{2} w^{2}(t)-\frac{M}{r(t)} \geq \frac{1}{2} w^{2}(0)-\frac{M}{r(0)}
$$

and

$$
\frac{1}{2} w^{2}(t) \geq \frac{1}{2} w^{2}(0)-\frac{M}{r(0)}
$$

on any time interval on which $w(t)$ remains non-negative. Now let $w_{-}>0$ be an initial lower bound for the radial velocities of the particles in the ensemble, $r_{-}>0$ an initial lower bound for their distance from the origin, and assume that

$$
W_{-}:=\frac{1}{2} w_{-}^{2}-\frac{M}{r_{-}}>0 .
$$

Then as long as a particle is moving outward,

$$
w(t)>W_{-}, r(t)>r_{-}+W_{-} t .
$$

But this implies that all the particles keep moving outward for all future time. For the Vlasov-Poisson system global existence and uniqueness of smooth solutions has been established for general initial data [6, 4, but the above argument can easily be extended to yield global existence for spherically symmetric initial data as specified above.

For the spherically symmetric Einstein-Vlasov system we find a substitute for the pseudo-energy $\frac{1}{2} w^{2}-\frac{M}{r}$ used above, but when taking its derivative along a particle trajectory it turns out to be important to use suitable coordinates on spacetime, namely maximal-areal coordinates, in order to see that under suitable circumstances this quantity does not decrease along radially outgoing trajectories. Once this is shown a bootstrap argument implies that all the particles keep moving radially outward on the future directed maximal interval of existence of the solution. We can then use a result from [10] to conclude that the solution is global in the time coordinate used, and the obtained control of the metric quantities turns out to be sufficient to establish future geodesic completeness. 


\section{The Einstein-Vlasov system}

We consider the system in the spherically symmetric, asymptotically flat case and write the metric in the following form:

$$
d s^{2}=-\left(\alpha^{2}-a^{2} \beta^{2}\right) d t^{2}+2 a^{2} \beta d t d r+a^{2} d r^{2}+r^{2}\left(d \theta^{2}+\sin ^{2} \theta d \phi^{2}\right) .
$$

Here the metric coefficients $\alpha, \beta$, and $a$ depend on $t \in \mathbb{R}$ and $r \geq 0, \alpha$ and $a$ are positive, and the polar angles $\theta \in[0, \pi]$ and $\phi \in[0,2 \pi]$ parameterize the unit sphere. The radial coordinate $r$ is thus the area radius. Let $K_{b}^{a}$ be the second fundamental form and define

$$
\kappa=K_{\theta}^{\theta}=\frac{\beta}{r \alpha} .
$$

By imposing the maximal gauge condition, which means that each hypersurface of constant $t$ has vanishing mean curvature, we obtain the following field equations where subscripts denote partial derivatives with respect to $r$ or $t$ :

$$
\begin{aligned}
a_{r} & =\frac{3}{2} a^{3} r \kappa^{2}+4 \pi r a^{3} \rho+\frac{a}{2 r}\left(1-a^{2}\right), \\
\kappa_{r} & =-\frac{3}{r} \kappa-4 \pi a \jmath, \\
a_{t} & =2 \alpha a \kappa+(a \beta)_{r}, \\
\alpha_{r r} & =\alpha_{r}\left(\frac{a_{r}}{a}-\frac{2}{r}\right)+\frac{2 \alpha}{r^{2}}\left(2 r \frac{a_{r}}{a}+a^{2}-1\right)+4 \pi a^{2} \alpha(S-3 \rho) .
\end{aligned}
$$

The Vlasov equation takes the form

$$
\partial_{t} f+\left(\frac{\alpha w}{a E}-\beta\right) \partial_{r} f+\left(-\frac{\alpha_{r} E}{a}-2 \alpha \kappa w+\frac{\alpha L}{a r^{3} E}\right) \partial_{w} f=0
$$

where

$$
E=E(r, w, L)=\sqrt{1+w^{2}+L / r^{2}} .
$$

The variables $w$ and $L$ can be thought of as the momentum in the radial direction and the square of the angular momentum respectively, see [7] for more details. The matter quantities which appear in the field equations are 
defined by

$$
\begin{aligned}
\rho(t, r) & =\frac{\pi}{r^{2}} \int_{-\infty}^{\infty} \int_{0}^{\infty} E f(t, r, w, L) d L d w, \\
\jmath(t, r) & =\frac{\pi}{r^{2}} \int_{-\infty}^{\infty} \int_{0}^{\infty} w f(t, r, w, L) d L d w \\
S(t, r) & =\frac{\pi}{r^{2}} \int_{-\infty}^{\infty} \int_{0}^{\infty} \frac{w^{2}+L / r^{2}}{E} f(t, r, w, L) d L d w .
\end{aligned}
$$

We impose the following boundary conditions which ensure asymptotic flatness and a regular center:

$$
a(t, 0)=a(t, \infty)=\alpha(t, \infty)=1 .
$$

The equations (2.2)-(2.10) together with the relation (2.1) and the boundary conditions (2.11) constitute the Einstein-Vlasov system for a spherically symmetric, asymptotically flat spacetime in maximal-areal coordinates.

First we note that the phase space density $f$ is constant along solutions of the characteristic system

$$
\begin{aligned}
\dot{r} & =\frac{\alpha(\tau, r) w}{a(\tau, r) E}-\beta(\tau, r), \\
\dot{w} & =-\frac{\alpha_{r}(\tau, r) E}{a(\tau, r)}-2 \alpha(\tau, r) \kappa(\tau, r) w+\frac{\alpha(\tau, r) L}{a(\tau, r) r^{3} E}, \\
\dot{L} & =0
\end{aligned}
$$

of the Vlasov equation. If $\tau \mapsto(R, W, L)(\tau, t, r, w, L)$ denotes the solution of the characteristic system with $(R, W, L)(t, t, r, w, L)=(r, w, L)$ then

$$
f(t, r, w, L)=\stackrel{\circ}{f}((R, W, L)(0, t, r, w, L)),
$$

with $\stackrel{\circ}{f}=f_{\mid t=0}$ the initial data for $f$. In particular, $f \geq 0$ provided this is true for the initial data as we will assume throughout. Next we notice that (2.3) can be rewritten as

$$
\left(r^{3} \kappa\right)_{r}=-4 \pi r^{3} a \jmath
$$

and upon integration

$$
\kappa(t, r)=-\frac{4 \pi}{r^{3}} \int_{0}^{r} a(t, s) \jmath(t, s) s^{3} d s .
$$


The Hawking mass $m$ is given by

$$
m=\frac{r}{2}\left(1+\frac{\beta^{2}}{\alpha^{2}}-\frac{1}{a^{2}}\right) .
$$

We also introduce the quantity

$$
\mu=\frac{r}{2}\left(1-\frac{1}{a^{2}}\right)
$$

and note that by (2.2), $\mu$ can be expressed as

$$
\mu(t, r)=\int_{0}^{r}\left(4 \pi \rho(t, s)+\frac{3}{2} \kappa^{2}(t, s)\right) s^{2} d s .
$$

Assuming that the matter is compactly supported initially and hence also for later times, (2.15) implies that $\kappa(t, r) \sim r^{-3}$ for $r$ large. Hence the limits as $r$ tends to $\infty$ of $m$ and $\mu$ are equal, so that the ADM mass $M$ is

$$
M=\int_{0}^{\infty}\left(4 \pi \rho(t, r)+\frac{3}{2} \kappa^{2}(t, r)\right) r^{2} d r .
$$

The ADM mass is conserved, and $\mu(t, r) \leq M$.

Finally we notice that using (2.2) the second order equation (2.5) can be rewritten in the form

$$
\left(\frac{r^{2}}{a} \alpha_{r}\right)_{r}=4 \pi r^{2} a \alpha(\rho+S)+6 r^{2} a \alpha \kappa^{2}
$$

and upon integration,

$$
\alpha_{r}(t, r)=\frac{a(t, r)}{r^{2}} \int_{0}^{r}\left(4 \pi \alpha a(\rho+S)+6 a \alpha \kappa^{2}\right) s^{2} d s .
$$

Thus $\alpha$ is monotonically increasing outwards, and from (2.11) it follows that

$$
0<\alpha \leq 1
$$

A direct computation using (2.5), (2.2), (2.17), and (2.18) implies that

$$
\left(\frac{r^{2}}{a^{2}} \alpha_{r}-\alpha \mu\right)_{r}=4 \pi r^{2} S \alpha+\frac{9}{2} r^{2} \kappa^{2} \alpha-4 \pi r^{3} \rho \alpha_{r}-\frac{3}{2} r^{3} \kappa^{2} \alpha_{r} .
$$


With the boundary conditions $(\alpha \mu)_{\mid r=0}=0=\left(r^{2} a^{-2} \alpha_{r}\right)_{\mid r=0}$ this yields the identity

$$
\frac{r^{2}}{a^{2}(t, r)} \alpha_{r}(t, r)=(\alpha \mu)(t, r)+\int_{0}^{r}\left[4 \pi S \alpha+\frac{9}{2} \kappa^{2} \alpha-4 \pi s \rho \alpha_{r}-\frac{3}{2} s \kappa^{2} \alpha_{r}\right] s^{2} d s,
$$

which turns out to be very convenient in what follows.

\section{The main result}

Let $\stackrel{\circ}{f}$ be some initial data of ADM mass $M$ such that

$$
\operatorname{supp} \stackrel{f}{f}] r_{-}, r_{+}\left[\times\left[w_{-}, \infty\left[\times\left[0, L_{+}\right] .\right.\right.\right.
$$

We wish to specify these parameters in such a way that for the corresponding solution of the Einstein-Vlasov system all particles have radial velocity $w>$ $W_{-}$with an additional parameter $W_{-}>0$. To this end we abbreviate

$$
\xi=\frac{2 M}{r_{-}}, \eta=\frac{W_{-}}{\sqrt{1+W_{-}^{2}+L_{+} / r_{-}^{2}}},
$$

and we require that the above parameters satisfy the following conditions:

$$
\begin{gathered}
\left(2 \min \left\{\frac{r_{-}}{r_{+}}, \frac{1-3 \xi}{\sqrt{1-\xi}+\xi / 2} \eta\right\}-1-\frac{9}{4} \frac{\xi}{1-\xi}-\frac{3}{2} \frac{\xi}{\sqrt{1-\xi}}-\frac{L_{+}}{W_{-} r_{-}^{2}}\right) W_{-}^{2} \\
-\left(1+\frac{L_{+}}{W_{-} r_{-}^{2}}+\frac{9}{4} \frac{\xi}{1-\xi}\right)\left(1+\frac{L_{+}}{r_{-}^{2}}\right)-\frac{L_{+}}{r_{-}^{2}} \frac{1}{1-\xi}>0
\end{gathered}
$$

and

$$
w_{-}^{2}>\frac{1}{1-\xi}\left(W_{-}^{2}+\xi\left(1+\frac{L_{+}}{r_{-}^{2}}\right)\right) .
$$

After stating our main result we will show that there exist parameters satisfying these conditions, and that there exist initial data with such parameters.

Theorem Suppose that $0<r_{-}<r_{+}, w_{-}>0, L_{+}>0, W_{-}>0$, and $M>0$ satisfy the conditions (3.2) and (3.3), and consider a solution $f$ on a maximal existence interval $\left[0, T\right.$ [ such that the initial data $\stackrel{\circ}{f}=f_{\mid t=0}$ is compactly supported, smooth, non-negative, has ADM mass $M$, and satisfies

$$
\operatorname{supp} \stackrel{\circ}{f} \subset] r_{-}, r_{+}\left[\times\left[w_{-}, \infty\left[\times\left[0, L_{+}\right] .\right.\right.\right.
$$


Then $T=\infty$, and the resulting spacetime is future geodesically complete.

The proof of this theorem is given in Section 4 below.

To show that parameters $\xi, r_{ \pm}, w_{-}, L_{+}, W_{-}$exist which satisfy (3.2) and (3.3) we proceed as follows. Let

$$
D(\xi, \eta)=2 \frac{1-3 \xi}{\sqrt{1-\xi}+\xi / 2} \eta-1-\frac{9}{4} \frac{\xi}{1-\xi}-\frac{3}{2} \frac{\xi}{\sqrt{1-\xi}}, \quad \xi \in[0,1[, \eta>0 .
$$

Since $D(0,1)=1$ and $D(1 / 3,1)<0$ there exists some $\left.\xi^{*} \in\right] 0,1 / 3[$ such that $D(\xi, 1)>0$ for $\xi \in\left[0, \xi^{*}\left[\right.\right.$ and $D\left(\xi^{*}, 1\right)=0$; numerically, $\xi^{*} \approx 0.0994$. So firstly we assume that

$$
\xi<\xi^{*} \text {. }
$$

Secondly, we choose $r_{+}>r_{-}>0$ such that

$$
2 \frac{r_{-}}{r_{+}}>1+\frac{9}{4} \frac{\xi}{1-\xi}+\frac{3}{2} \frac{\xi}{\sqrt{1-\xi}}
$$

where we notice that the right hand side is increasing in $\xi$ and its value for $\xi=\xi^{*}$ is $\approx 1.405$ so that the inequality can be satisfied with $r_{+}>r_{-}>0$. Now we fix $L_{+}>0$ arbitrarily. Since $\eta \rightarrow 1$ as $W_{-} \rightarrow \infty$ we can choose $W_{-}>0$ sufficiently large so that

$$
D(\xi, \eta)-\frac{L_{+}}{W_{-} r_{-}^{2}}>0
$$

and

$$
2 \frac{r_{-}}{r_{+}}>1+\frac{9}{4} \frac{\xi}{1-\xi}+\frac{3}{2} \frac{\xi}{\sqrt{1-\xi}}+\frac{L_{+}}{W_{-} r_{-}^{2}} .
$$

Then the factor multiplying $W_{-}^{2}$ in the condition (3.2) is positive, and by further increasing $W_{-}$if necessary condition (3.2) is satisfied. Finally, we take $w_{-}>0$ sufficiently large so that (3.3) holds as well.

Let us now fix a set of parameters $\xi, r_{ \pm}, L_{+}, w_{-}, W_{-}$such that (3.2) and (3.3) are satisfied. We want to argue that an initial condition with these parameter values does exist. In order to do so we fix some compactly supported, smooth function $\stackrel{\circ}{g}$ such that

$$
\operatorname{supp} \stackrel{\circ}{ } \subset] r_{-}, r_{+}\left[\times\left[w_{-}, \infty\left[\times\left[0, L_{+}\right]\right.\right.\right.
$$

and we consider $\stackrel{\circ}{f}=A \stackrel{\circ}{g}$ for some amplitude $A>0$. Quantities induced by $\stackrel{\circ}{f}$ will be denoted by the corresponding superscript, and they depend on $A$. By 
continuity, we can make sure that $\stackrel{a}{a}(r) \leq 2$ for all $A>0$ sufficiently small; the dependence of the initial data for the metric quantities on the initial matter terms and hence on $\stackrel{\circ}{f}$ will be studied in [3]. Now let

$$
I=\int_{0}^{\infty} 4 \pi \stackrel{\circ}{(s)} s^{2} d s
$$

Then by (2.15),

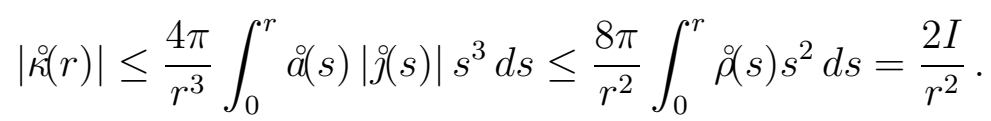

Since $\kappa(r)=0$ for $r \leq r_{-}$this implies that

$$
I \leq M=\int_{r_{-}}^{\infty}\left(4 \pi \stackrel{\circ}{(}(r)+\frac{3}{2} \stackrel{\circ}{ }^{2}(r)\right) r^{2} d r \leq I+\frac{6 I^{2}}{r_{-}} .
$$

Since the quantity $I$ depends linearly on the amplitude $A, 2 M(\stackrel{\circ}{f}) / r_{-}=\xi<$ $\xi^{*}$ for $A>0$ sufficiently small, and arguing again by continuity any value of $\xi \in] 0, \xi^{*}[$ should be realized by adjusting $A$ properly. Since $f$ has the same support as $\stackrel{\circ}{g}$, all conditions required in the theorem then hold for $\stackrel{\circ}{f}$.

\section{Proof of the theorem}

Step 1: The bootstrap argument.

Our first and crucial step in the proof of the theorem is a bootstrap argument which shows that the condition $w>W_{-}$which holds on the support of $f$ initially will persist on the maximal forward existence interval of the solution.

To begin with we show that for all $t \in\left[0, T\left[\right.\right.$ and $r \geq r_{-}$,

$$
a(t, r)<a_{+}=\frac{1}{\sqrt{1-\xi}} \text { and } \alpha(t, r)>\alpha_{-}=\frac{1-3 \xi}{1-\xi}
$$

eventually we shall see that these bounds hold for all $r \geq 0$. We recall that $\xi=2 M / r_{-}$. By (2.17),

$$
\frac{1}{a^{2}(t, r)}=1-\frac{2 \mu(t, r)}{r}>1-\frac{2 M}{r_{-}}=1-\xi
$$


for $r \geq r_{-}$; the inequality is strict because the solution is non-trivial and hence $\kappa$ does not vanish identically. By (2.20), (2.21), and (2.19),

$$
\alpha_{r}(t, r) \leq \frac{a_{+}^{2}}{r^{2}} \int_{0}^{r}\left(4 \pi(\rho+S)+6 \kappa^{2}\right) s^{2} d s<\frac{4 a_{+}^{2} M}{r^{2}} .
$$

Since $\alpha(t, \infty)=1$, this implies that

$$
\alpha(t, r)>1-\frac{4 a_{+}^{2} M}{r_{-}}=\frac{1-3 \xi}{1-\xi} .
$$

Next we define for $t \in[0, \infty[$,

$$
\begin{aligned}
& R_{-}(t)=r_{-}+\frac{\alpha_{-}}{a_{+}} \frac{W_{-}}{\sqrt{1+W_{-}^{2}+L_{+} / r_{-}^{2}}} t \\
& R_{+}(t)=r_{+}+\left(1+\frac{a_{+} M}{r_{-}}\right) t
\end{aligned}
$$

as well as

$$
\begin{aligned}
\gamma & =\min _{t \in[0, \infty[} \frac{R_{-}(t)}{R_{+}(t)}=\min \left\{\frac{r_{-}}{r_{+}}, \frac{\alpha_{-}}{a_{+}\left(1+a_{+} M / r_{-}\right)} \frac{W_{-}}{\sqrt{1+W_{-}^{2}+L_{+} / r_{-}^{2}}}\right\} \\
& =\min \left\{\frac{r_{-}}{r_{+}}, \frac{1-3 \xi}{\sqrt{1-\xi}+\xi / 2} \eta\right\},
\end{aligned}
$$

where we recall the abbreviations in (3.1) and (4.1). Let $\left[0, t_{0}[\right.$ denote the maximal time interval such that for $t \in\left[0, t_{0}[\right.$ and $(r, w, L) \in \operatorname{supp} f(t)$,

$$
R_{-}(t)<r<R_{+}(t), \quad w>W_{-} .
$$

By (3.3) and (3.4) this holds for $t=0$, and by continuity there is a maximal time interval $\left[0, t_{0}\left[\right.\right.$ with $t_{0}>0$ on which (4.6) persists. Suppose that $t_{0} \in$ ] $0, T\left[\right.$. Then we must have equality at $t=t_{0}$ in at least one of the inequalities in (4.6). But in the following part of the proof we show that (4.6) persists on $\left[0, t_{0}\right]$. Thus necessarily $t_{0}=T$.

Consider a characteristic such that $f(t, r(t), w(t), L)>0$. Denoting the parameter along the curve by $t$ it is calculated that

$$
\begin{aligned}
\frac{d}{d t}\left[\left(1-\frac{2 M}{r}\right) E^{2}\right]= & -2\left(1-\frac{2 M}{r}\right) \frac{\alpha_{r}}{a} E w-4 \frac{\beta}{r} w^{2}+6 \frac{M}{r^{2}} \beta w^{2} \\
& +2 \frac{L}{r^{3}} \beta-6 \frac{M}{r^{4}} \beta L+2 \frac{M}{r^{2}} \frac{\alpha}{a} E w-2 \frac{M}{r^{2}} \beta
\end{aligned}
$$


and we want to show that this quantity remains positive on the time interval $\left[0, t_{0}[\right.$. By (2.9) and (4.6) $), \jmath \geq 0$ for $t \in\left[0, t_{0}[\right.$. Hence (2.1) and (2.15) lead to the estimate

$$
\beta(t, r)=-\frac{\alpha(t, r)}{r^{2}} \int_{0}^{r} 4 \pi a(t, s) \jmath(t, s) s^{3} d s \leq 0
$$

and we can drop the fifth and seventh term in (4.7). Using the estimate

$$
\frac{1}{a^{2}(t, r)}=1-\frac{2 \mu(t, r)}{r} \geq 1-\frac{2 M}{r}
$$

and the fact that $\alpha_{r} \geq 0$, cf. (2.20), we find that

$$
\frac{d}{d t}\left[\left(1-\frac{2 M}{r}\right) E^{2}\right] \geq-2 \frac{\alpha_{r}}{a^{3}} E w-4 \frac{\beta}{r} w^{2}+\frac{2 \beta}{r^{2}}\left(3 M w^{2}+\frac{L}{r}\right)+2 \frac{M}{r^{2}} \frac{\alpha}{a} E w .
$$

By (2.22) and in view of $\alpha(t, s) \leq \alpha(t, r)$,

$$
\alpha_{r}(t, r) \leq \frac{M}{r^{2}}\left(\alpha a^{2}\right)(t, r)+\frac{\left(\alpha a^{2}\right)(t, r)}{r^{2}} \int_{0}^{r}\left(4 \pi S+\frac{9}{2} \kappa^{2}\right) s^{2} d s .
$$

Inserting this into (4.9) and using $a \geq 1$ and (4.8),

$$
\begin{aligned}
\frac{d}{d t}\left[\left(1-\frac{2 M}{r}\right) E^{2}\right] \geq \frac{2 \alpha}{r^{2}} & {\left[\frac{2 w^{2}}{r} \int_{0}^{r} 4 \pi j s^{3} d s-E w \int_{0}^{r}\left(4 \pi S+\frac{9}{2} \kappa^{2}\right) s^{2} d s\right.} \\
& \left.-\left(3 M w^{2}+\frac{L}{r}\right) \frac{1}{r^{2}} \int_{0}^{r} 4 \pi a j s^{3} d s\right]
\end{aligned}
$$

notice that the first term coming from the above estimate for $\alpha_{r}$ is exactly cancelled by the last term in (4.9), which is crucial. Now the estimates $w^{2} / E \leq w$ and $E \geq 1$ imply that

$$
\begin{aligned}
S(t, r) & \leq \jmath(t, r)+\frac{\pi}{r^{4}} \int_{-\infty}^{\infty} \int_{0}^{\infty} \frac{L}{E} f(t, w, r, L) d L d w \\
& \leq \jmath(t, r)+\frac{\pi}{r^{4}} \frac{L_{+}}{W_{-}} \int_{-\infty}^{\infty} \int_{0}^{\infty} w f(t, w, r, L) d L d w \\
& \leq\left(1+\frac{L_{+}}{W_{-}} \frac{1}{r^{2}}\right) \jmath(t, r) .
\end{aligned}
$$


By (2.15), $\kappa(t, r)=0$ for $r \leq R_{-}(t)$, and

$$
|\kappa(t, r)| \leq a_{+} \frac{4 \pi}{r^{2}} \int_{0}^{r} \jmath(t, s) s^{2} d s \leq a_{+} \frac{4 \pi}{r^{2}} \int_{0}^{r} \rho(t, s) s^{2} d s \leq \frac{a_{+} M}{r^{2}} .
$$

Let

$$
J(t, r)=\int_{0}^{r} 4 \pi \jmath(t, s) s^{2} d s \geq 0 .
$$

Then using the fact that $J$ is increasing in $r$ and $R_{-}(t) \geq r_{-}$for $t \in\left[0, t_{0}[\right.$,

$$
\int_{0}^{r} \kappa^{2} s^{2} d s \leq a_{+}^{2} M \int_{R_{-}(t)}^{r} \frac{1}{s^{2}}\left(\int_{0}^{s} 4 \pi j \tau^{2} d \tau\right) d s \leq \frac{a_{+}^{2} M}{R_{-}(t)} J \leq \frac{a_{+}^{2} M}{r_{-}} J .
$$

By the definition of $\gamma$, see (4.5),

$$
\frac{1}{r} \int_{0}^{r} 4 \pi \jmath(t, s) s^{3} d s \geq \gamma J(t, r) .
$$

We insert the estimates (4.11), (4.13), and (4.14) into (4.10) and use the fact that $r \geq R_{-}(t) \geq r_{-}$along the characteristic under consideration to obtain the estimate

$$
\begin{array}{r}
\frac{d}{d t}\left[\left(1-\frac{2 M}{r}\right) E^{2}\right] \geq \frac{2 \alpha}{r^{2}} J\left[2 \gamma w^{2}-\left(1+\frac{L_{+}}{W_{-}} \frac{1}{r_{-}^{2}}\right) E w-\frac{9}{2} \frac{a_{+}^{2} M}{r_{-}} E w\right. \\
\left.-\left(3 M w^{2}+\frac{L_{+}}{r_{-}}\right) \frac{a_{+}}{r_{-}}\right]
\end{array}
$$

Due to the estimate $E w \leq E^{2}=1+w^{2}+L / r^{2}$ this implies that

$$
\begin{aligned}
\frac{d}{d t}\left[\left(1-\frac{2 M}{r}\right) E^{2}\right] \geq & \frac{2 \alpha}{r^{2}} J\left[\left(2 \gamma-1-\frac{9}{2} \frac{a_{+}^{2} M}{r_{-}}-\frac{L_{+}}{W_{-} r_{-}^{2}}-\frac{3 a_{+} M}{r_{-}}\right) w^{2}\right. \\
& \left.-\left(1+\frac{L_{+}}{W_{-} r_{-}^{2}}+\frac{9}{2} \frac{a_{+}^{2} M}{r_{-}}\right)\left(1+\frac{L_{+}}{r_{-}^{2}}\right)-\frac{L_{+} a_{+}}{r_{-}^{2}}\right] .
\end{aligned}
$$

If we recall the definition of $\gamma$ in (4.5) we see that the condition (3.2) implies that the factor multiplying $w^{2}$ in the right hand side of this estimate is positive. Hence the estimate is still true if we replace $w$ by $W_{-}$, since by assumption $w^{2}>W_{-}^{2}$ on the time interval $\left[0, t_{0}[\right.$. Again recalling the condition (3.2) we conclude that

$$
\frac{d}{d t}\left[\left(1-\frac{2 M}{r}\right) E^{2}\right]>0
$$


as long as $t \in\left[0, t_{0}[\right.$. Hence for any characteristic in supp $f$ on this time interval,

$$
\left(1-\frac{2 M}{r(t)}\right)\left(1+w^{2}(t)+\frac{L}{r^{2}(t)}\right) \geq\left(1-\frac{2 M}{r(0)}\right)\left(1+w^{2}(0)+\frac{L}{r^{2}(0)}\right) .
$$

Thus

$$
w^{2}(t)+\frac{L}{r^{2}(t)} \geq\left(1-\frac{2 M}{r(0)}\right)\left(w^{2}(0)+\frac{L}{r^{2}(0)}\right)-\frac{2 M}{r(0)} .
$$

Since $\dot{r} \geq 0, r(t) \geq r(0)>r_{-}$. Together with $w(0) \geq w_{-}$this implies that

$$
\begin{aligned}
w^{2}(t) & \geq\left(1-\frac{2 M}{r_{-}}\right) w_{-}^{2}-\frac{2 M}{r_{-}}\left(1+\frac{L_{+}}{r_{-}^{2}}\right) \\
& =(1-\xi) w_{-}^{2}-\xi\left(1+\frac{L_{+}}{r_{-}^{2}}\right)>W_{-}^{2},
\end{aligned}
$$

the latter because of (3.3) . Therefore $w(t)>W_{-}$for $t \in\left[0, t_{0}\right]$. From (4.8) it follows that

$$
\beta(t, r) \geq-\frac{a_{+}}{r} \int_{0}^{r} 4 \pi j s^{2} d s \geq-\frac{a_{+} M}{r_{-}} .
$$

Thus by (2.12),

$$
\frac{\alpha_{-}}{a_{+}} \frac{W_{-}}{\sqrt{1+W_{-}^{2}+L_{+} / r_{-}^{2}}} \leq \dot{r} \leq 1+\frac{a_{+} M}{r_{-}} .
$$

Since $r(0) \in] r_{-}, r_{+}\left[\right.$, this implies that $R_{-}(t)<r(t)<R_{+}(t)$ for $t \in\left[0, t_{0}\right]$; recall (4.3) and (4.4). Therefore (4.6) holds on $\left[0, t_{0}\right]$, and $t_{0}=T$.

Step 2: Global existence in maximal-areal coordinates.

The next step in the proof of the theorem is to show that the estimates obtained in Step 1 on the maximal existence interval $[0, T$ [imply that $T=\infty$.

In [10, Thm. 2.1] sufficient conditions for global existence in maximalisotropic coordinates are obtained. It is straightforward to see that these conditions are also sufficient in our case, i.e., if we can show that the support of the momenta and the metric function $a$ do not blow up, then $T=\infty$ follows; the local well-posedness in maximal-areal coordinates and continuation of solutions will be studied more systematically in [3]. At points $(t, r, \theta, \phi)$ with $r<R_{-}(t)$ there is no matter. Hence by (2.15), (2.20), (2.2), and (2.11) this implies that

$$
a_{r}(t, r)=\alpha_{r}(t, r)=\kappa(t, r)=0 \text { for } r<R_{-}(t) .
$$


In particular the bounds in (4.1) hold for all $r \geq 0$ and $t \in[0, T[$. In view of the latter it is sufficient to verify that

$$
\sup \{E \mid(r, w, L) \in \operatorname{supp} f(t), t \in[0, T[\}<\infty .
$$

In what follows $C>0$ denotes a constant which only depends on the parameters specified in (3.2) and (3.3) and which may change from line to line. By (2.7) and (2.12)-(2.14) it follows that along characteristics,

$$
\dot{E}=-\frac{\alpha_{r} w}{a}-\frac{\alpha \kappa}{E}\left(2 w^{2}-\frac{L}{r^{2}}\right)
$$

Since by (4.6) and (4.3) $r(t) \geq R_{-}(t) \geq C(1+t)$ for any characteristic in supp $f$, (4.12) and (4.2) imply that

$$
|\kappa(t, r)|+\alpha_{r}(t, r) \leq \frac{C}{(1+t)^{2}}
$$

We point out that in this particular case when $w>0$ the first term of the right hand side of (4.17) can be dropped since it is non-positive but this more general estimate will be needed later. Since $\alpha \leq 1$ and $a \geq 1$ we thus obtain the estimate

$$
|\dot{E}| \leq C \frac{E}{(1+t)^{2}}
$$

and Gronwall's lemma implies the desired bound (4.16). Thus $T=\infty$.

Step 3: Future geodesic completeness.

In order to analyze this question polar coordinates such as we use on the spatial $t=$ const surfaces are not convenient, and we introduce new coordinates

$$
x^{0}=t, x^{1}=r \sin \theta \cos \phi, x^{2}=r \sin \theta \sin \phi, x^{3}=r \cos \theta,
$$

which can be thought of as the corresponding Cartesian coordinates. In these coordinates the metric becomes

$$
g_{00}=-\alpha^{2}+a^{2} \beta^{2}, g_{0 i}=a^{2} \beta \frac{x_{i}}{r}, g_{i j}=\delta_{i j}+\left(a^{2}-1\right) \frac{x_{i} x_{j}}{r^{2}},
$$

where Latin indices $i, j$ run from 1 to 3 and $x_{i}=\delta_{i j} x^{j}$. Let us now consider an arbitrary future directed, time-like or null geodesic, i.e., a solution $\left(x^{\gamma}(s), p^{\gamma}(s)\right)$ of the geodesic equations

$$
\begin{aligned}
& \frac{d x^{\gamma}}{d s}=p^{\gamma}, \\
& \frac{d p^{\gamma}}{d s}=-\Gamma_{\delta \epsilon}^{\gamma} p^{\delta} p^{\epsilon},
\end{aligned}
$$


where Greek indices $\gamma, \delta, \epsilon$ run from 0 to $3, \Gamma_{\delta \epsilon}^{\gamma}$ are the Christoffel symbols, and

$$
p^{0}>0, g_{\gamma \delta} p^{\gamma} p^{\delta}=-m^{2} \leq 0 ;
$$

$m^{2}$ is conserved along any geodesic, and $p^{0}$ cannot change sign. Such a geodesic exists on a maximally extended interval $\left[0, s_{+}[\right.$, and future geodesic completeness means that $s_{+}=\infty$ for all such geodesics.

The following relations between the variables $r, w, L$, and $p^{\gamma}$ hold:

$$
\begin{aligned}
E & =\alpha p^{0}, \\
w & =\left(\frac{x_{i} p^{i}}{r}+\beta p^{0}\right) a, \\
\frac{L}{r^{2}} & =\delta_{i j} p^{i} p^{j}-\left(\frac{x_{i} p^{i}}{r}\right)^{2},
\end{aligned}
$$

where we now re-define

$$
E=E(r, w, L)=\sqrt{m^{2}+w^{2}+L / r^{2}} .
$$

That we had $m=1$ before means that our system describes an ensemble of particles of rest mass 1 , but for geodesic completeness we have to consider any time-like or null geodesic, i.e., we have to allow any $m \geq 0$. Since $d t / d s=p^{0}>0$ we can re-parameterize the geodesic by coordinate time $t \in\left[0, t_{+}[\right.$. Along the geodesic again (4.17) holds. By (4.15), $\dot{E}=0$ as long as $r \leq R_{-}(t)$ so that also (4.18) holds along the geodesic, and $E \leq C$ for some constant $C$. Since by (4.1) $\alpha$ is bounded from below, $p^{0}$ remains bounded on $\left[0, t_{+}\left[\right.\right.$. Since both $a$ and $\beta$ are bounded as well also the $p^{i}$ remain bounded, and hence $t_{+}=\infty$. But since $d t / d s=p^{0}$ is bounded, this implies that $s_{+}=\infty$, and the proof of the theorem is complete.

\section{References}

[1] H. Andréasson, The Einstein-Vlasov System/Kinetic Theory, Living Rev. Relativ. 8 (2005).

[2] H. Andréasson, G. Rein, A numerical investigation of the stability of steady states and critical phenomena for the spherically symmetric Einstein-Vlasov system. Class. Quantum Grav. 23, 36593677 (2006). 
[3] H. Andréasson, M. Kunze, G. Rein, The spherically symmetric Einstein-Vlasov system in maximal areal coordinates. In preparation.

[4] P.-L. Lions, B. Perthame, Propagation of moments and regularity for the 3-dimensional Vlasov-Poisson system, Invent. Math. 105, 415-430 (1991).

[5] I. Olabarrieta, M. W. Choptuik, Critical phenomena at the threshold of black hole formation for collisionless matter in spherical symmetry, Phys. Rev. D. 65, 024007 (2002).

[6] K. Pfaffelmoser, Global classical solutions of the VlasovPoisson system in three dimensions for general initial data, J. Differential Equations 95, 281-303 (1992).

[7] G. ReIn, The Vlasov-Einstein system with surface symmetry, Habilitationsschrift, Munich (1995).

[8] G. Rein, A. D. Rendall, Global existence of solutions of the spherically symmetric Vlasov-Einstein system with small initial data, Commun. Math. Phys. 150, 561-583 (1992). Erratum: Commun. Math. Phys. 176, 475-478 (1996).

[9] G. Rein, A. D. Rendall, J. Schaeffer, Critical collapse of collisionless matter - a numerical investigation, Phys. Rev. D 58, 044007 (1998).

[10] A. D. Rendall, An introduction to the Einstein-Vlasov system, Banach Center Publ. 41, 35-68 (1997). 